\section{A cavitary pneumonia caused by leptotrichia species in an immunocompetent patient}

\author{
Tze Shien Lo ${ }^{1,2}$ \\ 'Section of Infectious Disease, VA \\ Medical Center; ${ }^{2}$ Department of Internal \\ Medicine, University of North Dakota \\ School of Medicine and Health Sciences, \\ Fargo, ND, USA
}

\section{Abstract}

Leptotrichia species (LS) is an anaerobic Gram negative bacillus in the Bacteroidaceae family and part of the normal human oral flora. It is rarely pathogenic, but occasionally causes diseases in immunocompromised hosts. I am reporting a case of cavitary pneumonia caused by LS in an immunocompetent host.

\section{Case Report}

An 81-year-old diabetic male came to our hospital to seek medical attention for 2 weeks of productive cough, fever and chills, and a 10 Kg weight loss. Physical examination revealed a fatigued-looking man with mild respiratory distress. Except for bilateral crackles in both lungs, more on the right side; the remainder of the physical examination was unremarkable, including the oral cavity. Complete blood count revealed mild anemia, however, the patient did not have leukopenia/neutropenia. A chest $\mathrm{x}$ ray showed a right lung infiltrate; thus, community-acquired pneumonia (CAP) was suspected and the patient was admitted for treatment of CAP.

The patient was started on intravenous gatifloxacin $400 \mathrm{mg}$ daily. However, the patient's symptoms continued to deteriorate while on gatifloxacin. A chest computed tomography (CT) done on hospital day 3 , showed a right upper lobe cavitary lesion (Figure 1). Because of the cavitary lesion, pulmonary diseases more complicated than CAP (such as lung cancer or vasculitis involving the lung) were suspected. The patient underwent a bronchoscopy on hospital day 4, which was reported as unremarkable. The bronchial washing cytology examination did not reveal malignant cells, but it showed scattered elongated bacilli (Figure 2). Within 24 hours the bronchial washing culture grew the same Gram-negative bacillus, methicillin-sensitive staphylococcus aureus, viridans streptococcus and Group B streptococcus. The Gram-negative bacillus was eventually identified as LS at the North Dakota State
Laboratory (Figure 3). Blood cultures were drawn - once before starting antibiotics and three more times during treatment; however, none of these cultures grew organisms. Because of the clinical unresponsiveness to gatifloxacin, on hospital day 6 , the antibiotic was changed to intravenous vancomycin $1.5 \mathrm{~g}$ daily and piperacillin-tazobactam (pip-tazo) $3.375 \mathrm{~g}$ every 8 hours.

The patient's symptoms improved while on vancomycin and pip-tazo. The pneumonia was suspected to be caused by one of the above Gram positive cocci and the LS was considered an oral contaminant; therefore, after a 9-day course of vancomycin and pip-tazo, vancomycin was continued but pip-tazo was discontinued on hospital day 15. On hospital day 19, after reconsidering LS a remotely possible pathogen of the pneumonia, we added oral amoxicillin $500 \mathrm{mg} /$ clavulanate $125 \mathrm{mg}$ every 8 hours to the vancomycin regimen. While on vancomycin and amoxicillin-clavulanate, the patient began to have spiked fever, rigors and increased dyspnea. On hospital day 23, a chest radiograph indicated worsening of the pneumonia. We then strongly suspected LS was the etiologic agent causing the pneumonia. We discontinued vancomycin and amoxicillin-clavulanate and reinitiated pip-tazo. The patient improved rapidly after this change in antibiotics. Pip-tazo was continued for 4 weeks, followed by 2 weeks of intravenous aqueous penicillin $\mathrm{G} 2$ million units every 8 hours. The patient continued to do well and has had no recurrence of pneumonia to date.

\section{Discussion}

LS is an anaerobic Gram-negative bacillus that resides in the oral cavity and usually does not cause disease. However, this bacterium can occasionally cause systemic infection in immunocompromised patients. Bacteremia, endocarditis, and hepatic abscess caused by Leptotrichia buccalis have been reported in patients with neutropenia, hematologic malignancies and in bone marrow transplant recipients. ${ }^{1-8}$ Mucosal disruptions such as oral mucositis, esophageal ulcers, or diverticulitis are possible risk factors for infected patients.

By Gram stain alone, LS can be confused with other oral commensals, such as the elongated Gram negative Fusobacteria sp, Capnocytophaga sp or even with the Gram positive Lactobacillus sp as LS can sometimes be Gram-variable. The LS in this case report was identified by Gram stain and phenotypic analysis, but not by gene sequencing because the North Dakota State Laboratory was not equipped to perform the test.

Only two cases of pneumonia caused by LS
Correspondence: Tze Shien Lo, Section of Infectious Disease (11C-I), VA Medical Center, 2101 Elm Street, Fargo, ND 58102, USA Tel. +1.701.232.3241 - Fax: +1.701.293.4145. E-mail: tzeshien.lo@med.und.edu

Key words: Leptotrichia sp, cavitary pneumonia, immunocompetent patient.

Received for publication: 31 October 2011. Revision received: 31 January 2012. Accepted for publication: 4 February 2012.

This work is licensed under a Creative Commons Attribution NonCommercial 3.0 License (CC BYNC 3.0).

(C) Copyright Tze Shien Lo, 2012

Licensee PAGEPress, Italy

Infectious Disease Reports 2012; 4:e24

doi:10.4081/idr.2012.e24

have been reported in the literature. One case is cavitary pneumonia with bacteremia caused by $L$. buccalis in an immunocompromised host. ${ }^{9}$ The other case is a severe pneumonia caused by LS in an apparently immunocompetent male patient. ${ }^{10}$ Our patient has type 2 diabetes mellitus, but he is not immunocompro-

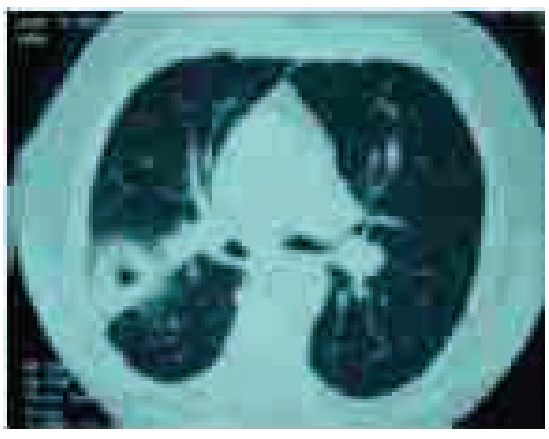

Figure 1. Computed tomography-scan of the chest shows a cavitary lesion in the right upper lobe.

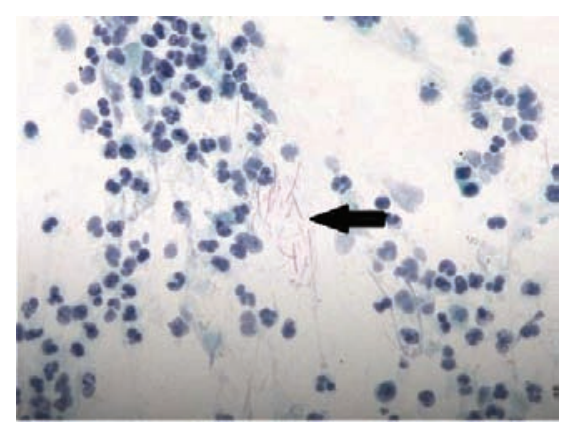

Figure 2. Papanicolaou stain of the bronchial washing reveals the elongated bacilli (arrow). Magnification $400 \times$. 


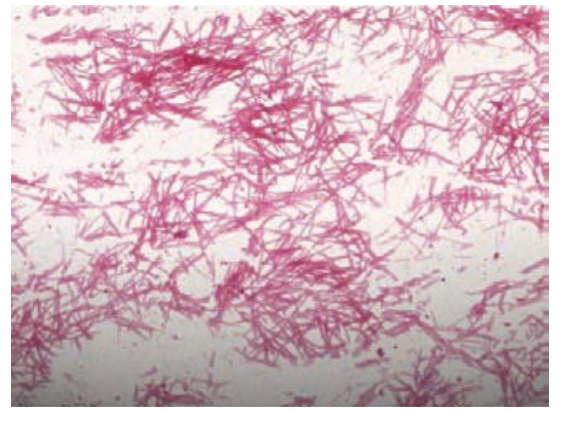

Figure 3. Gram stain of the sputum culture shows Leptotrichia sp. Magnification $400 \times$.

mised from other underlying diseases or from immunosuppressive agents; therefore, I believe this is the first case report of cavitary pneumonia caused by LS in an immunocompetent patient. Although the patient had good dental hygiene without dental caries, gingivitis, or oral mucosa lesions; the oral cavity could still be the portal of entry in this patient's pneumonia. Pneumonia caused by LS could be underreported because LS is usually considered a normal oral flora and is difficult to identify. Therefore; if LS grows from bronchoalveolar lavage or lung tissue, a clinician should consider it one of the etiologic agents causing pneumonia.

Due to the rarity of human infection caused by LS, the duration of treatment for pneumonia caused by this bacterium is not clear. In our case, because the pneumonia recurred after we administered pip-tazo for only 9 days, we aggressively gave the patient 4 weeks of piptazo followed by 2 weeks of intravenous aqueous penicillin $\mathrm{G}$.

Although there are no Clinical and Laboratory Standards Institute (CLSI) breakpoints for LS, penicillins are considered the drugs of choice. Thus, the patient continued to be clinically stable while on either pip-tazo or penicillin G. We postulate that the pneumonia recurred while on amoxicillin-clavulanate was likely due to the oral agent at a subtherapeutic level in the lung tissue. Therefore, we do not advocate treating pneumonia caused by LS with an oral $\beta$-lactam antibiotic.

\section{References}

1. Bhally HS, Lema C, Romagnoli M, et al. Leptotrichia buccalis bacteremia in two patients with acute myelogenous leukemia. Anaerobe 2005;11:350-3.

2. Weinberer $\mathrm{M}, \mathrm{Wu} \mathrm{T}$, Rubin $\mathrm{M}$, et al. Leptotrichia buccalis bacteremia in patients with cancer: report of four cases and review. Rev Infect Dis 1991;13:201-6.

3. Vemelen K, Mertens I, Thomas J, et al. Bacteremia with Leptotrichia buccalis: report of a case and review of the litera- ture. Acta Clin Belg 1996;51:265-70.

4. Schwartz DN, Schable B, Tenover FC, Miller RA. Leptotrichia buccalis bacteremia in patients treated in a single bone marrow transplant unit. Clin Infect Dis 1995;20:762-7.

5. Reig M, Baquero F, Garcia-Campello M, Loza E. Leptotrichia buccalis bacteremia in neutropenic children. J Clin Microbiol 1985;22:320-1.

6. Kohler JL, Raoult D, Gallais H, et al. Septicemia due to Leptotrichia buccalis in an immuno-suppressed patient. Sem Hop 1982;58:1767-8. [Article in French].

7. Hammann R, Iwand A, Brachmann J, et al. Endocarditis caused by a Leptotrichia buccalis-like bacterium in a patient with prosthetic aortic valve. Eur J Clin Microbiol Infect Dis 1993;12:280-2.

8. Duperval R, Beland S, Marcoux JA. Infective endocarditis due to Leptotrichia buccalis: a case report. Can Med Assoc J 1984;130:422-4.

9. Morgenstein AA, Citron DM, Orisek B, Finegold SM. Serious infection with Leptotrichia buccalis. Report of a case and review of the literature. Am J Med 1980;69:782-5.

10. Kawanami T, Fukuda K, Yatera K, et al. Severe pneumonia with Leptotrichia sp. Detected predominantly in bronchoalveolar lavage fluid by use of $16 \mathrm{~S}$ rRNA gene sequencing analysis. J Clin Microbiol 2009;47:496-8. 